\title{
Polarization-gradient cooling in a strong doughnut-mode dipole potential
}

\author{
S. Kuppens, * M. Rauner, M. Schiffer, K. Sengstock, and W. Ertmer \\ Institut für Quantenoptik, Universität Hannover, Welfengarten 1, 30167 Hannover, Germany \\ F. E. van Dorsselaer and G. Nienhuis \\ Huygens Laboratorium, Rijksuniversiteit Leiden, Postbus 9504, 2300 RA Leiden, The Netherlands
}

(Received 20 January 1998)

\begin{abstract}
We present experimental and theoretical studies of polarization-gradient cooling of metastable neon atoms confined to the dark center of a $\mathrm{TEM}_{01}^{*}$ (doughnut) mode. A slow beam of neon atoms is guided and focused inside a blue-detuned and focused doughnut-mode laser beam to a spot size below $10 \mu \mathrm{m}$. The transverse motion inside this doughnut mode is cooled by means of two-dimensional optical molasses. We observed non-Gaussian two-component velocity distributions of which the cold component has a width of down to three recoil velocities. These results are found to be in qualitative agreement with a quantum Monte Carlo simulation of cooling in one dimension in the presence of an external light-shift potential. For the simulation we apply the recently developed technique of quantum-state diffusion with adaptive noise. [S1050-2947(98)03710-X]
\end{abstract}

PACS number(s): 32.80.Pj, 42.50.Lc

\section{INTRODUCTION}

In the effort to prepare dense and cold samples of atoms for high precision measurements and the observation of quantum collective effects all-optical methods receive more and more interest [1]. Especially, the low photon scattering rate of far-off-resonance dipole traps is very promising in this respect. A prerequisite for obtaining low temperatures and high densities in such traps is an efficient cooling mechanism, which preferentially cools the atoms right down to the vibrational ground state of the trap. Recent efforts to cool inside dipole traps include schemes such as Raman cooling [2] and evaporative cooling [3]. We focus on the realization of a scheme for continuous loading of a bluedetuned doughnut-mode dipole trap. The doughnut mode is a good candidate for trapping atoms because the atoms can be confined to the dark center of the mode, which strongly reduces the spontaneous scattering of photons. Recently, we demonstrated the guiding and focusing properties of the doughnut mode [4,5]. In this article we discuss detailed experimental and theoretical studies on the two-dimensional (2D) cooling of neon atoms in a blue-detuned doughnut mode.

Our main objective is to demonstrate polarizationgradient cooling inside the doughnut-mode atom guide. A key question to be addressed is to what extent the light shifts induced by the confining doughnut mode laser beam alter the cooling process. Although the atoms are confined to the dark region of the mode, their spatial distribution may partly reach into regions where the light shift resulting from the doughnut mode is not negligible compared to the light shifts induced by the cooling light. Experimentally, we obtained momentum spreads as small as three recoil velocities inside the doughnut mode, being only slightly larger than the mini-

\footnotetext{
*Present address: JILA and Department of Physics, University of Colorado and National Bureau of Standards, Boulder, CO 803090440 .
}

mum spread achieved with the same cooling configuration in free space. Higher cooling light intensities are required for the cooling to become effective in stronger doughnut-mode potentials; the light shifts induced by the cooling light have to exceed those resulting from the doughnut mode.

The influence of additional light shifts induced by the doughnut mode on the cooling process was investigated by performing an additional theoretical study. This was done using the recently developed method of quantum trajectories with adaptive noise [6]. In contrast to about 1000 quantum trajectories needed in conventional quantum trajectory methods the adaptive method requires only 20 trajectories to obtain a good ensemble average. The application to our experimental configuration clearly demonstrates the potential of this calculation method. Although the simulations are based on one-dimensional cooling they qualitatively agree with the experimental results on the characteristic dependence of the final momentum distribution on the various parameters. The time constants for the cooling process obtained from the simulation are expected to be slightly smaller as compared to the actual cooling in 2D.

The paper is organized as follows. In Sec. II and Sec. III we describe the experimental configuration and the obtained results, respectively. In Secs. IV and V a one-dimensional configuration is considered, as well as numerical simulations thereof. Finally the comparison between the experiment and the numerical simulations is summarized in Sec. VI.

\section{PHYSICAL FRAMEWORK}

In this section we introduce the general physical framework to describe the guiding and cooling process in the doughnut-mode dipole potential used throughout the following sections.

The experiments are performed with metastable neon atoms in the $3 s[3 / 2]_{2}$ state. The guiding and cooling laser lights both act on the same electronic transition of $\mathrm{Ne}^{*}$, $3 s[3 / 2]_{2} \rightarrow 3 p[5 / 2]_{3}$ at $\lambda=640.2 \mathrm{~nm}$. This cycling transition has a natural linewidth of $\Gamma=1 / \tau=52 \times 10^{6} \mathrm{~s}^{-1}$, and a satu- 
ration intensity of $I_{\text {sat }}=41.3 \mathrm{~W} / \mathrm{m}^{2}$.

The setup of the experiment for the guiding and focusing of an atom beam is schematically depicted in Fig. 1. A cold beam of metastable neon atoms is made to axially overlap with a doughnut-mode laser beam by sending the atoms through a hole in a mirror. The $z$ axis is chosen to coincide with the mutual axes of the atom beam and the guiding laser beam, and is in the following denoted as longitudinal direction.

Throughout this paper we denote by the term doughnut mode the $\mathrm{TEM}_{01}^{*}$ Laguerre-Gaussian mode with azimuthal mode number $l=1$ and radial mode number $p=0$ [7]. In the work presented here we concentrate on the light shifts induced by the doughnut mode, which are proportional to the intensity profile of the mode [8],

$$
I_{d}(\mathbf{r})=\frac{4 P_{d}}{\pi w_{d}^{4}(z)}\left(x^{2}+y^{2}\right) e^{-2\left(x^{2}+y^{2}\right) / w_{d}^{2}(z)} .
$$

Here $w_{d}(z)=w_{0} \sqrt{1+z^{2} / z_{R}^{2}}$ is the $z$-dependent beam radius, $w_{0}$ is the beam waist, $z_{R}$ is the Rayleigh range of the beam, and $P_{d}$ is the power.

For a discussion of the principle we first consider a twolevel atom with level spacing $\hbar \omega_{0}$ inside such a doughnut mode, which is detuned from the resonance by an amount $\Delta_{d}=\omega_{d}-\omega_{0}$. In the absence of saturation one can adiabatically eliminate the upper level. The remaining effect of the interaction with the laser light is a shift (ac Stark shift) of the energy of the lower state proportional to the laser intensity,

$$
U_{d}(\mathbf{r})=\frac{\hbar \Omega_{d}^{2}(\mathbf{r})}{4 \Delta_{d}},
$$

where $\Omega_{d}(\mathbf{r})$ is the Rabi frequency,

$$
\Omega_{d}^{2}(\mathbf{r})=\frac{\Gamma^{2}}{2} \frac{I_{d}(\mathbf{r})}{I_{\text {sat }}} .
$$

Due to the intensity distribution of the doughnut mode the shift is a position dependent potential. For blue detuning $\left(\Delta_{d}>0\right)$ this potential is repulsive and gives rise to a force pointing towards low intensity, resulting in a confinement of the atom near the axis of the doughnut mode beam.

As the atoms move towards the focus of the doughnut mode the potential gets narrower and the maximum potential well depth $U_{\max }(z)$ increases,

$$
U_{\max }(z)=\frac{\hbar \Gamma^{2}}{4 \Delta_{d}} \frac{P_{d}}{\pi w_{d}^{2}(z)} \frac{1}{I_{\text {sat }}} e^{-1} .
$$

In this way the injected atom beam is focused. Due to the transverse spatial compression the transverse kinetic energy of the atoms increases as they approach the focus of the doughnut mode.

Near the axis of the doughnut mode $\left(x, y \ll w_{d}\right)$ the potential is harmonic. This leads to equidistant vibrational energy levels when the transverse motion of the atoms is described quantum mechanically. The level spacing is given by

$$
\hbar \Omega_{\mathrm{vib}}(z)=\hbar \sqrt{\frac{4 e U_{\max }(z)}{m w_{d}^{2}(z)}},
$$

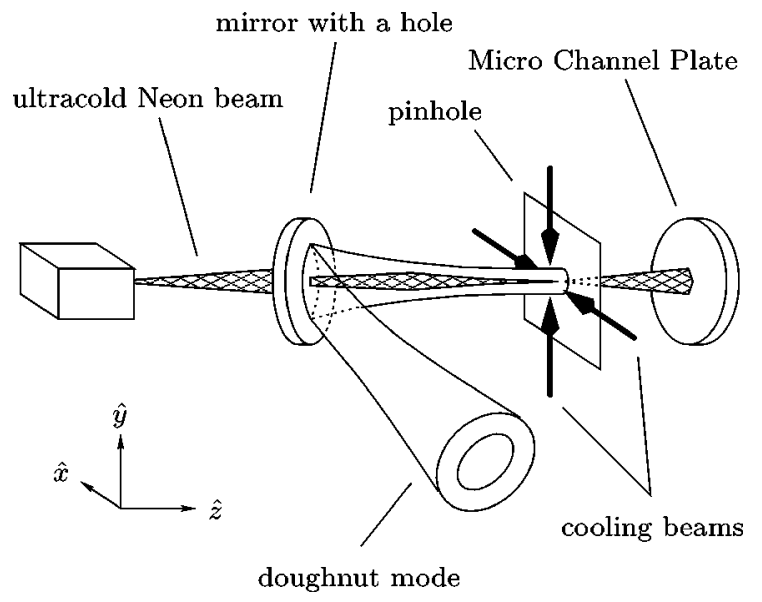

FIG. 1. Schematic diagram of the experimental setup used for guiding and sub-Doppler cooling of atoms in a blue-detuned doughnut mode.

where $m$ is the atomic mass. With the setup discussed here, vibrational level spacings well above one photon recoil energy, $E_{\text {rec }}=(\hbar k)^{2} / 2 m$, can be achieved in the focus of a doughnut mode. For typical experimental parameters, $P_{d}$ $=350 \mathrm{~mW}, \Delta_{d}=2 \pi \times 10 \mathrm{GHz}$, and $w_{0}=40 \mu \mathrm{m}$, the potential contains about 7000 vibrational levels at a spacing of $\hbar \Omega_{\mathrm{vib}}=2.5 E_{\mathrm{rec}}$.

Since polarization-gradient cooling leads to temperatures of a few recoil energies, it is expected to cool a substantial fraction of the atoms to low-lying vibrational states. For the case of a $J_{g}=1 / 2 \rightarrow J_{e}=3 / 2$ transition this expectation was confirmed by a quantum Monte Carlo simulation in Ref. [9].

To study this process experimentally the transverse motion of the optically guided atoms is cooled by a twodimensional optical molasses extending $8 \mathrm{~mm}$ along the $z$ axis in the focus of the doughnut mode, just in front of the pinhole (see Fig. 1).

Polarization-gradient cooling depends crucially on the Zeeman structure of the transition. For a correct description of the experiment the angular momenta of the lower and upper levels of the 640-nm transition $\left(J_{g}=2 \rightarrow J_{e}=3\right)$ have to be taken into account. In Sec. IV a more general description of the atom-field interaction is presented that accounts for the Zeeman structure and for the fact that both the guiding and cooling light act on the same cycling transition at $640 \mathrm{~nm}$. The actual setup and experimental results will be discussed in the next section.

\section{EXPERIMENTAL RESULTS}

\section{A. Setup}

The ultracold beam of metastable neon atoms is generated by Zeeman slowing a collimated thermal beam from a liquidnitrogen-cooled dc discharge source with supersonic expansion. After the Zeeman slowing, the beam is further cooled, compressed, and deflected by a two-dimensional magnetooptical molasses (2D-MOM) [10,11]. Due to a large deflection angle of $68^{\circ}$ the resulting atom beam is free of UV photons and contains only metastable atoms in the required state. For a detailed description of our beam preparation we refer to Refs. $[12,13]$. The final beam has a sub-Doppler 
transverse root-mean-square ( $\mathrm{rms}$ ) velocity spread of $7 \mathrm{~cm} / \mathrm{s}$ (2.3 recoil velocities $v_{\text {rec }}$ ) and a longitudinal velocity of 28 $\mathrm{m} / \mathrm{s}$ with a $4 \mathrm{~m} / \mathrm{s}$ rms spread.

In the experiment the cold beam of metastable neon atoms is injected into a blue detuned doughnut mode laser beam through a hole in a mirror that is placed at an angle of $45^{\circ}$ with the atom beam (see Fig. 1). The hole in the 2-mm-thick mirror has a diameter of $60 \mu \mathrm{m}$. At this mirror the laser beam has a radius $w_{d}$ of typically $500 \mu \mathrm{m}$, which is much larger than the hole in the mirror in order to keep its effect on the doughnut mode profile as small as possible. Due to the spatial extension of the atom beam $1 \%$ of the atoms pass through the $60-\mu \mathrm{m}$ hole, corresponding to a flux of 1.4 $\times 10^{6}$ atoms/s injected into the doughnut-mode laser beam [14]. The doughnut mode reaches a focus $\left(w_{0}=45 \mu \mathrm{m}\right)$ at a $16-\mu \mathrm{m}$-diameter pinhole, positioned $14 \mathrm{~cm}$ downstream from the mirror.

The spatial distribution of the atoms is detected $20 \mathrm{~cm}$ behind the pinhole with a microchannel plate (MCP) with phosphor screen and intensified CCD camera. Since the pinhole forms a point source of atoms to very good approximation the 2D spatial distribution on the MCP is directly related to their velocity distribution. The resolution of our detection system is $0.5 v_{\text {rec }}$.

The doughnut-mode laser beam $\left(\mathrm{TEM}_{01}^{*}\right)$ is derived from the Gaussian $\left(\mathrm{TEM}_{00}\right)$ output beam of a commercial ring dye-laser by holographical means $[15,16]$. A detailed description of the technique to holographically generate pure $\mathrm{TEM}_{p l}^{*}$ modes will be published elsewhere [17]. Essentially, the hologram is a superposition of a spiral phase plate and a blazed phase grating. About $40 \%$ of the incoming Gaussian mode is transformed into a doughnut mode in the first diffraction order of the grating. Starting with a Gaussian input beam of $1 \mathrm{~W}$ this generates $400 \mathrm{~mW}$ of doughnut-mode power available for the atom guiding. The doughnut mode is linearly polarized. The laser frequency is stabilized to a line width of $3 \mathrm{MHz}$. The long term drift of approximately 10 $\mathrm{MHz}$ per hour is negligible compared to the detuning of $10-1000 \mathrm{GHz}$ used for atom guiding. The beam pointing was actively stabilized to be better than $1.6 \pm 0.3 \mu \mathrm{m} \mathrm{rms}$ deviation in the focal plane of the doughnut mode.

The light for the polarization-gradient cooling was obtained from a second frequency stabilized ring dye-laser. Long term stability of $2 \mathrm{MHz}$ was achieved by applying Doppler-free absorption spectroscopy on the thermal atom beam.

Up to a power of $50 \mathrm{~mW}$ of cooling light was available. The cooling light beam waists were $w_{y}=2.9 \mathrm{~mm}$ and $w_{z}$ $=9.2 \mathrm{~mm}$, in the vertical and longitudinal direction, respectively. Two knife edges blocked the wings of the intensity distribution to obtain a nearly square intensity profile, $8 \mathrm{~mm}$ wide along the $z$ axis. Throughout the following the cooling light intensity will often be expressed in terms of the dimensionless single beam saturation parameter, given by

$$
s=\frac{\Omega_{c}^{2}}{2\left(\Gamma^{2} / 4+\Delta_{c}^{2}\right)} .
$$

For a detuning $\Delta_{c}$ of $-9 \Gamma$ and a power of $50 \mathrm{~mW}$ the saturation parameter $s$ is 0.09 , where the Clebsch-Gordan coefficient was taken to be unity.
The 2D optical molasses consists of two crossed standing waves generated from the above-described 8-mm-wide laser beam (see Fig. 1). Two different configurations of cooling light polarizations were investigated, which we denote by $\pi_{x}-\pi_{y}$ and $\sigma_{x}-\sigma_{y}$, respectively. The configuration $\pi_{x}-\pi_{y}$ consists of two perpendicular standing waves with linear polarizations in the plane of the beams. The ellipticity was measured to be less than $10 \%$. Alternatively, in the $\sigma_{x}-\sigma_{y}$ configuration each pair of beams consists of two oppositely circularly polarized beams. The ratio of the linear polarization components along $x$ and $y$ was 8:11. The magnetic fields in the region of the cooling zone were compensated to below $10 \mu \mathrm{T}$.

\section{B. Guiding and focusing}

First we discuss the guiding and focusing properties of the doughnut mode without cooling. The spatial and velocity spread of the atoms depend on the doughnut-mode parameters, such as power, detuning, and waist. They set the initial conditions for the cooling process.

The spatial distribution of the atoms was measured by scanning the pinhole in the $x y$ plane. For a detuning of 100 $\mathrm{GHz}$ and a power of $300 \mathrm{~mW}$ the Gaussian width was 6.5 $\pm 2.4 \mu \mathrm{m}$ and the peak intensity was 6.4 $\times 10^{11}$ atoms s${ }^{-1} \mathrm{~cm}^{-2}$. In this case $60 \pm 10 \%$ of the atoms injected into the doughnut mode are guided. Increasing the detuning to a value of $300 \mathrm{GHz}$ at the same power, thus lowering $U_{\max }$, results in a broader distribution having a width of $14.4 \pm 2.4 \mu \mathrm{m}$, as expected.

More important for the cooling process is the width of the velocity distribution with which the atoms enter the cooling zone. As mentioned in Sec. II the compression of the spatial distribution leads to a simultaneous broadening of the transverse velocity distribution.

We measured 2D transverse velocity distributions of the atoms in the doughnut mode at the position of the pinhole (see Sec. III A) as a function of the depth $U_{\max }$ of the doughnut mode potential. For this measurement the power $P_{d}$ was kept fixed at $300 \mathrm{~mW}$ and the value of $U_{\max }$ was varied by varying the detuning $\Delta_{d} / 2 \pi$ between 20 and $1000 \mathrm{GHz}$. In Fig. 2 the Gaussian width of the measured velocity distribution is plotted as a function of $U_{\max }$. The data plotted in Fig. 2 clearly show that the width of the transverse velocity distribution increases with $U_{\max }$, as expected. Note that the width well exceeds the Doppler width of $10 \hbar k$ for large values of $U_{\max }$.

In summary, by increasing the value of $U_{\max }$ the initial spatial distribution before cooling is narrowed, whereas the velocity distribution becomes broader. These results concerning the guiding and focusing properties of the doughnut mode set the starting conditions for our investigation of polarization-gradient cooling inside the doughnut-mode dipole potential, which is discussed in Sec. III C.

\section{Cooling inside a blue-detuned doughnut mode}

Polarization-gradient cooling was studied as a function of the cooling light intensity for different doughnut-mode potential strengths and for the two distinct sets of polarizations of the cooling light: $\pi_{x}-\pi_{y}$ and $\sigma_{x}-\sigma_{y}$ as introduced in Sec. II. The distance between the mirror (see Fig. 1) and the pin- 


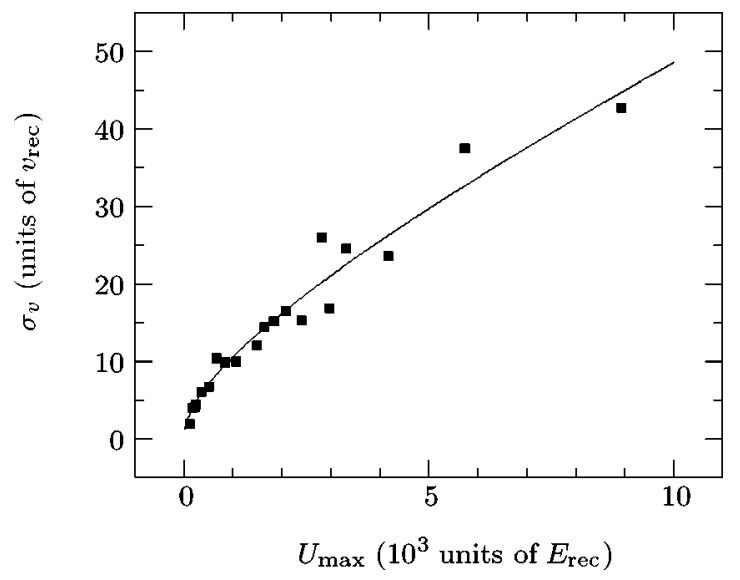

FIG. 2. Gaussian width of the velocity distribution without cooling as a function of the potential well depth $U_{\max }$ in the focal plane of the doughnut mode. The symbols denote the experimental results and the solid curve is a square root fit to the data.

hole was set to $10.5 \mathrm{~cm}$, keeping the focus of the doughnut mode in the plane of the pinhole. Unless otherwise stated the detuning of the cooling light will be $\Delta_{c}=-9 \Gamma$ throughout the following.

In Fig. 3 1D cross sections of the 2D velocity distribution are shown for different cooling light intensities. In this particular case the $\sigma_{x}-\sigma_{y}$ polarization configuration was used. The doughnut mode had a detuning of $246 \mathrm{GHz}$ and a power of $400 \mathrm{~mW}$, resulting in $U_{\max }=2033 E_{\text {rec }}$ in the focal plane.

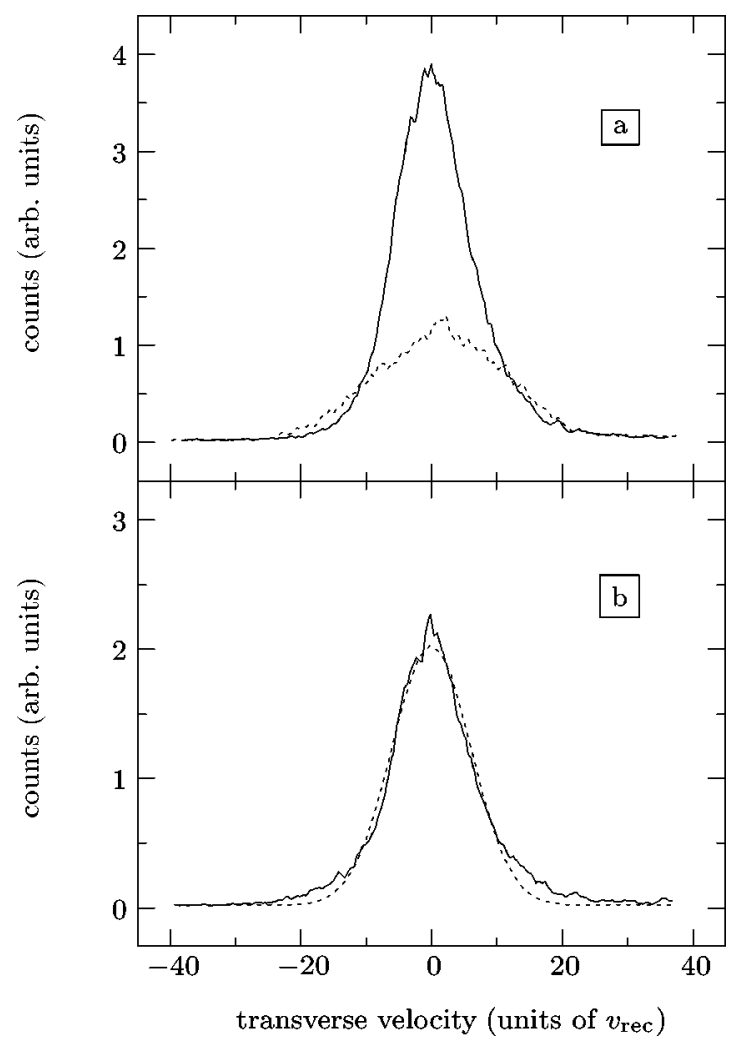

FIG. 3. One-dimensional cross section of the measured $2 \mathrm{D}$ velocity distribution for $\sigma_{x}-\sigma_{y}$ polarized cooling light. (a) Dashed curve is without cooling, and solid curve is $100 \mathrm{~mW} / \mathrm{cm}^{2}$ cooling light intensity; (b) solid curve is $40 \mathrm{~mW} / \mathrm{cm}^{2}$ cooling light intensity, and dashed curve is a Gaussian fit to the data.
Clearly, the width of the velocity distribution is reduced and simultaneously the height is increased due to the cooling. The total number of atoms in the full 2D distribution is conserved. In comparison to the Doppler limit for neon atoms, being $10 v_{\text {rec }}$ (at the optimal detuning of $-\Gamma / 2$ ), the width of the cooled distribution is more than a factor of 2 smaller, in this particular case. Within the experimental uncertainty the width of the spatial distribution was found to be the same with or without cooling. This was checked for different strengths of the doughnut-mode potential.

A characteristic feature of the cooling in our experiment is the fact that it leads to a non-Gaussian velocity distribution. This can be seen from a comparison between the solid and dashed curve in Fig. 3(b). Clearly, the dashed curve, which is a single Gaussian, only poorly fits the data. A much better fit is obtained by using a fitting function consisting of two Gaussians, a broad and a narrow one. The $\chi^{2}$ of the fit is then more than a factor of 5 smaller. This was observed for a large range of cooling light and doughnut-mode parameters. The fraction of atoms in the narrow component increases with increasing cooling light intensity. For strong doughnut modes $\left(U_{\max }<2500 E_{\text {rec }}\right)$ cooling is still observed but the two-component shape vanishes and the cooled velocity distribution becomes Gaussian.

We observe velocity distributions consisting of a broad pedestal, having the width of the uncooled distribution, and a narrow sub-Doppler peak on top of it also in the case of a vanishing doughnut mode guiding field. These shapes are a generic feature of transverse sub-Doppler cooling of atomic beams [18-21] where the interaction time with the cooling beams is relatively short and the cooling light intensity is high. The narrow peak is due to sub-Doppler cooling and the broad peak is the result of the Doppler cooling forces [21].

First we will focus on cooling in relatively weak doughnut-mode potentials, i.e., $U_{\max }>2500 E_{\text {rec }}$ where the two-component velocity distribution is observed. In Fig. 4 the widths of the two components are plotted as a function of the cooling light saturation parameter for both polarization configurations and different doughnut-mode strengths. It is remarkable that the widths of both components of the cooled distribution are rather insensitive to the cooling light intensity, irrespective of the polarizations chosen. However, the fraction of atoms in the cold (narrow) component increases with increasing intensity up to values of $70 \%$.

When comparing the two polarization configurations, $\sigma_{x}-\sigma_{y}$ and $\pi_{x}-\pi_{y}$, a slight difference in the intensity dependence of the width of the velocity distribution and the fraction of cold atoms is observed. In order to exclude a possible dependence on the initial width of the velocity distribution we compare the two data sets plotted in Fig. 4 measured for approximately equal values of $U_{\max }$ (1510 and 1376 units of $\left.E_{\text {rec }}\right)$, but for different cooling light polarization. In the case of $\sigma_{x}-\sigma_{y}$ polarization the fraction of cold atoms increases nearly linearly with intensity. For the $\pi_{x}-\pi_{y}$ polarization the increase of the fraction of cold atoms is smaller at low intensities. Above a threshold intensity of $10 \mathrm{~mW} / \mathrm{cm}^{2}$ it then rapidly increases to the same value obtained with $\sigma_{x}-\sigma_{y}$ polarization.

Theoretical calculations [22] of the cooling force in onedimensional polarization-gradient cooling for the $J=2 \rightarrow J$ $=3$ transition show that the friction coefficient in a $\operatorname{lin} \perp$ lin 


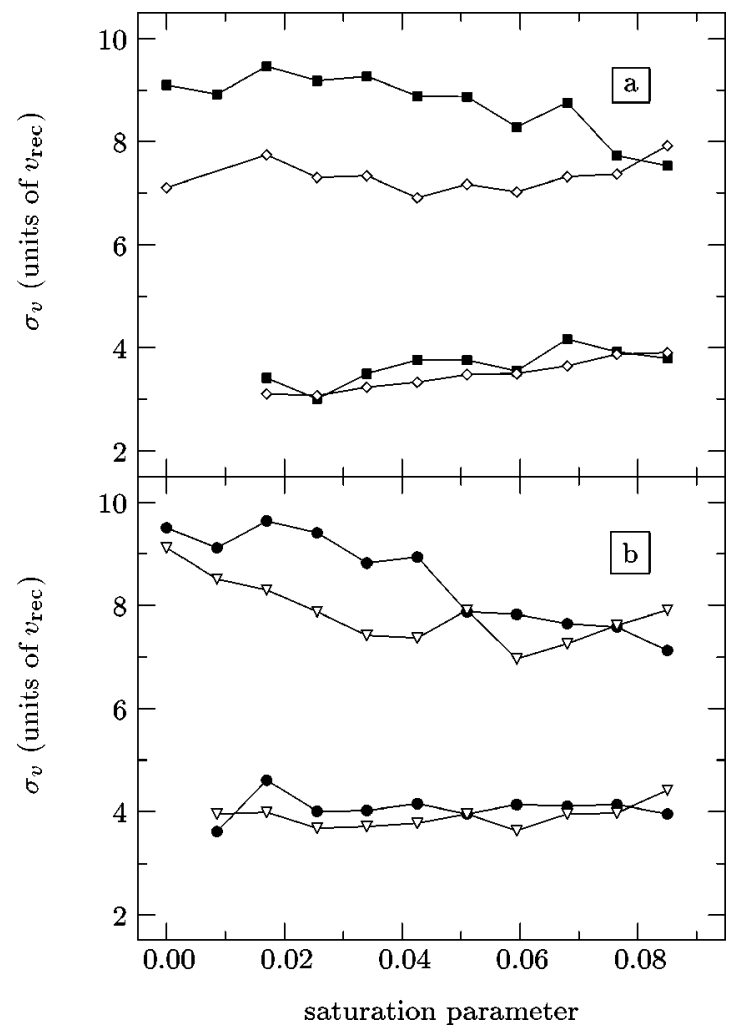

FIG. 4. Widths of the two Gaussian components of the velocity distribution in the focal plane of the doughnut mode for moderate values of $U_{\max }$ as a function of the cooling light saturation parameter. (a) $\pi_{x}-\pi_{y}$ polarized cooling light: squares: $\Delta_{d}=327 \mathrm{GHz}$, $P_{d}=360 \mathrm{~mW}, U_{\max }=1376 E_{\text {rec }}$; diamonds: $\Delta_{d}=463 \mathrm{GHz}, P_{d}$ $=390 \mathrm{~mW}, U_{\max }=1053 E_{\mathrm{rec}}$. (b) $\sigma_{x}-\sigma_{y}$ polarized cooling light: circles: $\Delta_{d}=246 \mathrm{GHz}, P_{d}=400 \mathrm{~mW}, U_{\max }=2033 E_{\mathrm{rec}}$; triangles: $\Delta_{d}=331 \mathrm{GHz}, P_{d}=400 \mathrm{~mW}, U_{\max }=1510 E_{\mathrm{rec}}$. The hot (upper) and cold (lower) component widths corresponding to the same parameter set are plotted with the same symbols.

configuration is larger than that of a $\sigma_{+}-\sigma_{-}$configuration, but the latter has a much larger capture range. This larger capture range may explain the more rapid increase of the fraction of cold atoms as a function of intensity in our twodimensional $\sigma_{x^{-}} \sigma_{y}$ cooling situation.

Also the intensity dependence of the width of the cold contribution to the velocity distribution slightly differ for the two polarization configurations, as can be inferred from Fig. 4 . For the $\pi_{x}-\pi_{y}$ polarization the width of the cold component increases from $3 v_{\text {rec }}$ to $4 v_{\text {rec }}$ with increasing intensity, whereas for the $\sigma_{x^{-}} \sigma_{y}$ polarization the width, within the accuracy of our measurements, remains constant at a value of $4 v_{\text {rec }}$ over the whole range of investigated intensities. The data in Fig. 4 also show that the final width of the cold component of the velocity distribution depends only weakly on the value of $U_{\max }$. As the upper curves in Fig. 4 show, the initial width before cooling depends on the value of $U_{\max }$. Polarization cooling also reduces the width of this hot background.

For a quantitative comparison we also investigated cooling with the $\pi_{x}-\pi_{y}$ configuration in the absence of the guiding doughnut mode laser beam and found similar twocomponent velocity distributions, with the fraction of cold atoms increasing with cooling light intensity. As observed in

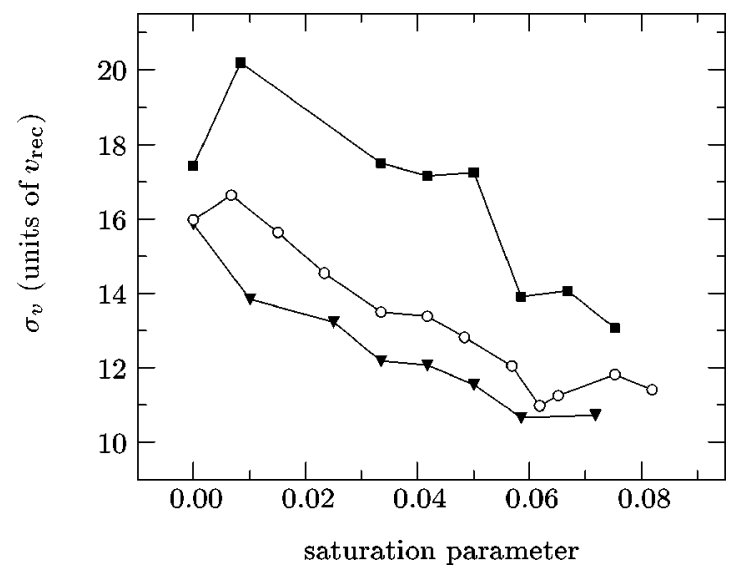

FIG. 5. Single-Gaussian width of the velocity distribution in the focal plane of the doughnut mode as a function of cooling light intensity for $U_{\max }>2500 E_{\mathrm{rec}}$ and for the cooling light polarization $\sigma_{x}-\sigma_{y}$. Squares: $\Delta_{d}=76 \mathrm{GHz}, P_{d}=355 \mathrm{~mW}, U_{\max }=5747 E_{\mathrm{rec}}$. Circles: $\Delta_{d}=103 \mathrm{GHz}, P_{d}=280 \mathrm{~mW}, U_{\max }=3400 E_{\text {rec }}$. Triangles: $\Delta_{d}=164 \mathrm{GHz}, P_{d}=375 \mathrm{~mW}, U_{\max }=2860 E_{\mathrm{rec}}$.

the situation where the doughnut mode was present also in free space the width of the cold component of the velocity distribution was found to be nearly independent of the cooling light intensity. The initial velocity spread being $15 v_{\text {rec }}$ and $7 v_{\text {rec }}$, the measured width of the cold component was $2.7 v_{\text {rec }}$ and $2.2 v_{\text {rec }}$, respectively.

Comparing the cooling inside the doughnut mode to cooling without the doughnut mode present, we observe that the typical two-component velocity distribution signature of the cooling process survives as long as the doughnut mode becomes not too strong (as illustrated below). However, the velocity width of the cold component is slightly larger, by about $1 v_{\text {rec }}$, when cooling is applied inside the doughnut mode for moderate values of $U_{\max }$. This shows that in this regime the presence of the doughnut mode has only a minor influence on the cooling process. The mechanism behind this increase of the velocity width is further discussed in Sec. V.

We now turn to the discussion of polarization gradient cooling in stronger doughnut-mode potentials, $U_{\max }$ $>2500 E_{\text {rec }}$, where the shape of the velocity distribution remains Gaussian during cooling. The widths of single Gaussian fits to the data are plotted in Fig. 5 as a function of the cooling light intensity. The initial widths are between $16.5 v_{\text {rec }}$ and $20 v_{\text {rec }}$ and decrease with increasing intensity to values between $10 v_{\text {rec }}$ and $13 v_{\text {rec }}$. Compared to cooling in a weak doughnut-mode potential the cooling behaves differently in two respects. The velocity distribution consists of a single Gaussian and the final velocity width after cooling remains larger than the small widths between $3 v_{\text {rec }}$ and $4 v_{\text {rec }}$ previously obtained in the weaker doughnut mode. In this case the time constant of the cooling process may be larger due to the presence of the strong doughnut-mode potential, which could explain the larger velocity spreads. On the other hand the change in the shape of the distribution indicates that not only the time constant of the cooling process is altered. The results of the simulations presented in Sec. V show that the larger velocity widths are not fully explained by a larger time constant. This point will be discussed in Sec. V. 


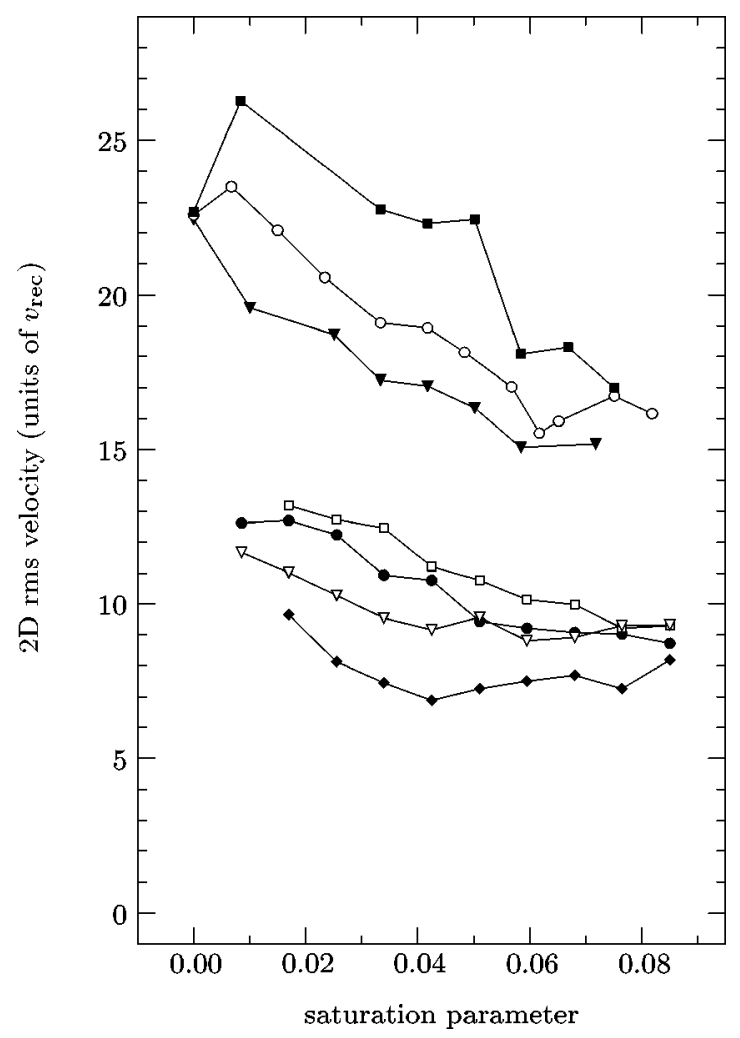

FIG. 6. Transverse rms velocity as a function of cooling light saturation parameter for weak and strong doughnut modes. The lower four curves correspond to the data in Fig. 4, and the upper three curves correspond to the data in Fig. 5. The value of $U_{\max }$ decreases in descending order of the curves as 5840, 3400, 2860, $2033,1510,1376$, and $1053 E_{\text {rec }}$.

For comparison of the two regimes we calculated the 2D rms velocity, $v_{\text {rms }}=\sqrt{\left\langle v_{x}^{2}+v_{y}^{2}\right\rangle}$, of the complete distribution from the fitting parameters of the data presented in Fig. 4 and Fig. 5 and plotted the result in Fig. 6, again as a function of the cooling light intensity. In both regimes the overall width of the transverse velocity distribution is reduced substantially. With increasing $U_{\max }$ the final temperature of cooling increases. As will be argued in detail in Sec. V, we interpret this as follows. With increasing cooling light intensity the light shifts induced by the cooling light increase. Since polarization gradient cooling depends crucially on these light shifts they have to exceed the light shifts imposed by the doughnut mode, which will happen at higher cooling intensities for stronger doughnut mode potentials.

In summary, we clearly demonstrated 2D polarizationgradient cooling of neon atoms confined to the dark center of a strong dipole potential formed by a doughnut mode laser beam. The velocity distribution was found to become nonGaussian upon cooling. The cold component of the resulting two-component velocity distribution had a width of $3 v_{\text {rec }}$ to $4 v_{\text {rec }}$ for $\pi_{x}-\pi_{y}$ polarization of the cooling light and $4 v_{\text {rec }}$ for $\sigma_{x^{-}} \sigma_{y}$ polarization. For both polarizations this width is nearly independent of the cooling light intensity but the fraction of cold atoms increases with intensity. Without the doughnut mode the same cooling setup resulted in a slightly smaller width of the velocity distribution of $2.2 v_{\text {rec }}$. By investigating the overall $\mathrm{rms}$ velocity of the cooled atoms we find that the end temperature reached increases with increas- ing strength of the doughnut mode potential. This will also be discussed further in Sec. V.

\section{THEORETICAL FRAMEWORK}

For the description of the atom-field interaction we adopt the formalism of Ref. [22]. We briefly summarize it here in order to fix the notation. Consider an atom at position $\mathbf{r}$ interacting with a plane traveling wave

$$
\mathbf{E}(\mathbf{r}, t)=\mathbf{E}_{0} \cos (\mathbf{k} \cdot \mathbf{r}-\omega t)
$$

of optical frequency $\omega$, wave vector $\mathbf{k}$, and amplitude $\mathbf{E}_{0}$. This field drives transitions between a ground state $|g\rangle$ of angular momentum $J_{g}$ and an upper state $|e\rangle$ of angular momentum $J_{e}$, whose energy separation is $\hbar \omega_{0}$. The interaction Hamiltonian in the dipole and rotating wave approximations is, in the rotating frame, expressed as

$$
H_{\mathrm{int}}(\mathbf{r})=\frac{\hbar \Omega}{2}\left[(\hat{\boldsymbol{\epsilon}} \cdot \mathbf{Q}) e^{i \mathbf{k} \cdot \mathbf{r}}+\text { H.c. }\right] \text {, }
$$

where $\hat{\boldsymbol{\epsilon}}$ is the polarization vector of the electric field. The strength of the coupling is given by the Rabi frequency $\Omega$ $=\Gamma \sqrt{I_{0} / 2 I_{\text {sat }}}$. The vectorial nature of the atomic dipoleelectric field interaction is described by $\hat{\boldsymbol{\epsilon}} \cdot \mathbf{Q}$. Here, $\mathbf{Q}$ is a reduced dimensionless dipole operator, which has the spherical components $Q_{\beta}=\hat{\mathbf{u}}_{\beta} \cdot \mathbf{Q}$, with

$$
\begin{gathered}
\hat{\mathbf{u}}_{-1}=(\hat{\mathbf{x}}-i \hat{\mathbf{y}}) / \sqrt{2}, \\
\hat{\mathbf{u}}_{0}=\hat{\mathbf{z}}, \\
\hat{\mathbf{u}}_{1}=-(\hat{\mathbf{x}}+i \hat{\mathbf{y}}) / \sqrt{2}
\end{gathered}
$$

the spherical unit vectors. The spherical components of $\mathbf{Q}$ are generalized raising operators transferring the ground state to the excited state; the matrix elements are Clebsch-Gordan coefficients, $\left\langle J_{e} M_{e}\left|Q_{\beta}\right| J_{g} M_{g}\right\rangle=\left\langle J_{e} M_{e} \mid J_{g} M_{g} ; 1 \beta\right\rangle$ for $\beta$ $=0, \pm 1$. Right $\sigma_{z}^{+}$and left $\sigma_{z}^{-}$circular polarization in the $x y$ plane correspond to $\beta=1$ and $\beta=-1$, respectively, and $\beta$ $=0$ describes linear $\pi_{z}$ polarization along the $z$ axis.

The doughnut mode guiding light field and the cooling light act on the same $J_{g}=2 \rightarrow J_{e}=3$ transition. In the effective Hamiltonian for the interaction of the atoms with the guiding doughnut laser beam only the light shifts of the ground and excited level are retained,

$$
H_{d}(\mathbf{r})=\frac{\hbar \Omega_{d}^{2}(\mathbf{r})}{4 \Delta_{d}}\left[\left(\hat{\boldsymbol{\epsilon}}_{d} \cdot \mathbf{Q}\right)^{\dagger}\left(\hat{\boldsymbol{\epsilon}}_{d} \cdot \mathbf{Q}\right)-\left(\hat{\boldsymbol{\epsilon}}_{d} \cdot \mathbf{Q}\right)\left(\hat{\boldsymbol{\epsilon}}_{d} \cdot \mathbf{Q}\right)^{\dagger}\right]
$$

where the Rabi frequency is given by Eq. (2.3). Optical pumping induced by the doughnut beam can be ignored in view of the inequality $\Gamma \ll \Omega_{d} \ll \Delta_{d}$, which holds for typical experimental parameters and distances of the atoms from the $z$ axis. In Table I the spherical components of the polarization vector are listed for linear and circular polarization in the $x y$ plane. Note that the excited state was not fully adiabatically eliminated. The first term in Eq. (4.4) probes the ground-state population whereas the second term probes the 
TABLE I. Possible doughnut beam polarizations. $\theta$ determines the orientation of linear polarization in the $x y$ plane.

\begin{tabular}{ccc}
\hline \hline$\beta$ & $\hat{\epsilon}_{d}=\pi_{x y}$ & $\hat{\epsilon}_{d}=\sigma_{z}^{+}$ \\
\hline+1 & $1 / \sqrt{2}$ & 1 \\
0 & 0 & 0 \\
-1 & $e^{i \theta / \sqrt{2}}$ & 0 \\
\hline \hline
\end{tabular}

excited-state population. Equation (4.4) is the generalization of Eq. (2.2) when the two levels involved have a Zeeman substructure. For positive (blue) detuning the Hamiltonian results in a repulsive potential confining the atoms in the central dark region of the doughnut mode.

The polarization gradient cooling depends on the light shifts as well as on the optical pumping induced by the laser light. The Hamiltonian for our 2D molasses is given by

$$
\begin{aligned}
H_{c}(\mathbf{r})= & -\hbar \Delta_{c} \mathbf{Q} \cdot \mathbf{Q}^{\dagger}+\frac{\hbar \Omega_{c}}{2}\left[\left(\hat{\boldsymbol{\epsilon}}_{+x} \cdot \mathbf{Q} e^{i k_{c} x}+\hat{\boldsymbol{\epsilon}}_{-x} \cdot \mathbf{Q} e^{-i k_{c} x}\right)\right. \\
& + \text { H.c. }+\left(\hat{\boldsymbol{\epsilon}}_{+y} \cdot \mathbf{Q} e^{i k_{c} y+i \phi}+\hat{\boldsymbol{\epsilon}}_{-y} \cdot \mathbf{Q} e^{-i k_{c} y-i \phi}\right) \\
& + \text { H.c. }]
\end{aligned}
$$

where $\phi$ is a possible time phase difference between the two pairs of beams. In the actual experiment the time phase $\phi$ is not stabilized. The phase fluctuations are on a much slower time scale than the atoms need to traverse the cooling light, so we can assume that each atom encounters one distinct phase. The first term in Eq. (4.5) describes the internal energy of the atom in the rotating frame and the second term describes the interaction. The spherical components of the polarization vectors $\hat{\boldsymbol{\epsilon}}_{+x}, \hat{\boldsymbol{\epsilon}}_{-x}, \hat{\boldsymbol{\epsilon}}_{+y}$, and $\hat{\boldsymbol{\epsilon}}_{-y}$ of the four traveling waves constituting the molasses are listed in Table II and Table III for the $\pi_{x^{-}} \pi_{y}$ and $\sigma_{x^{-}} \sigma_{y}$ polarization configuration, respectively.

The combined action of the guiding and cooling light is described by the total Hamiltonian

$$
H(\mathbf{r}, \mathbf{p})=\frac{\mathbf{p}^{2}}{2 m}+H_{d}(\mathbf{r})+H_{c}(\mathbf{r}),
$$

where $\mathbf{p}$ is the atomic momentum of the center-of-mass motion and $m$ is the atomic mass.

The dynamics of the atoms internal and external degrees of freedom are described by the following master equation for the density matrix $\rho(t)$ of the system,

TABLE II. Polarization vectors for a linearly polarized cooling configuration.

\begin{tabular}{lcc}
\hline \hline$\beta$ & $\hat{\epsilon}_{+x}=\hat{\epsilon}_{-x}=\pi_{y}$ & $\hat{\epsilon}_{+y}=\hat{\epsilon}_{-y}=\pi_{x}$ \\
\hline+1 & $1 / \sqrt{2}$ & $1 / \sqrt{2}$ \\
0 & 0 & 0 \\
-1 & $-1 / \sqrt{2}$ & $1 / \sqrt{2}$ \\
\hline \hline
\end{tabular}

TABLE III. Polarization vectors for a circularly polarized cooling configuration.

\begin{tabular}{lcccc}
\hline \hline \multicolumn{1}{c}{$\beta$} & $\hat{\boldsymbol{\epsilon}}_{+x}=\sigma_{x}^{+}$ & $\hat{\boldsymbol{\epsilon}}_{-x}=\sigma_{x}^{-}$ & $\hat{\boldsymbol{\epsilon}}_{+y}=\sigma_{y}^{+}$ & $\hat{\boldsymbol{\epsilon}}_{-y}=\sigma_{y}^{-}$ \\
\hline+1 & $-1 / 2$ & $1 / 2$ & $1 / 2$ & $1 / 2$ \\
0 & $1 / \sqrt{2}$ & $1 / \sqrt{2}$ & $-i / \sqrt{2}$ & $i / \sqrt{2}$ \\
-1 & $1 / 2$ & $-1 / 2$ & $1 / 2$ & $1 / 2$ \\
\hline \hline & \\
$\frac{d}{d t} \rho(t)=$ & $-\frac{i}{\hbar}[H(\mathbf{r}, \mathbf{p}), \rho(t)]-\frac{\Gamma}{2}\left[\mathbf{Q} \cdot \mathbf{Q}^{\dagger} \rho(t)+\rho(t) \mathbf{Q} \cdot \mathbf{Q}^{\dagger}\right.$ \\
& $-2 \int d \hat{\mathbf{u}} \sum_{\hat{\boldsymbol{\epsilon}} \perp \hat{\mathbf{u}}} g \hat{\boldsymbol{\epsilon}}(\hat{\mathbf{u}})(\hat{\boldsymbol{\epsilon}} \cdot \mathbf{Q})^{\dagger}$ \\
& $\times e^{-i k \hat{\mathbf{u}} \cdot \mathbf{x}} \rho(t)(\hat{\boldsymbol{\epsilon}} \cdot \mathbf{Q}) e^{i k \hat{\mathbf{u}} \cdot \mathbf{x}] .}$
\end{tabular}

The Hamiltonian part describes the coherent evolution. The remaining part describes the coupling to the reservoir of vacuum fluctuations. The first two terms within this coupling describe the coherent decrease of the excited-state amplitude. The last term describes the feeding of the ground state by spontaneous emission. It contains an integral over all possible directions and polarizations of the emitted photons, where $g_{\hat{\boldsymbol{\epsilon}}}(\hat{\mathbf{u}})$ is the probability distribution that a photon is emitted in the direction $\hat{\mathbf{u}}$ with a polarization $\hat{\boldsymbol{\epsilon}}$.

This sets the framework for the theoretical description of the combined guiding and cooling inside a blue-detuned doughnut mode. In Sec. V we will numerically simulate the evolution of $\rho(t)$ given by Eq. (4.7).

\section{NUMERICAL SIMULATIONS}

We describe both the internal state of the atoms as well as the translational degrees of freedom quantum mechanically. In order to numerically calculate the final temperatures as described by Eq. (4.7) for different cooling parameters we have to make a number of approximations due to the complexity of the system. The state of the system is simulated using the momentum eigenstates as basis. However, this makes the position dependence of the interaction hard to implement. The atoms move relatively slowly in the transverse direction while they are in the cooling zone, i.e., the guiding potential acts on a much longer scale. Hence we completely ignore the position dependence of the doughnut potential and we assume that each atom experiences a homogeneous light shift operator. We approximate Eq. (4.4) by

$$
H_{d}=U_{d}\left[\left(\hat{\boldsymbol{\epsilon}}_{d} \cdot \mathbf{Q}\right)^{\dagger}\left(\hat{\boldsymbol{\epsilon}}_{d} \cdot \mathbf{Q}\right)-\left(\hat{\boldsymbol{\epsilon}}_{d} \cdot \mathbf{Q}\right)\left(\hat{\boldsymbol{\epsilon}}_{d} \cdot \mathbf{Q}\right)^{\dagger}\right]
$$

where $U_{d}$ is the effective (average) light shift that the atoms experience, i.e., a certain fraction of the maximum light shift at the maximum of the doughnut potential. Note that within this approximate model the effect of the doughnut is fully described by this single parameter and the polarization of the doughnut beam. Although we have introduced a spatially uniform light shift operator we will refer to $U_{d}$ as doughnutmode light shift for the purpose of briefness. 
Since, for the interaction, the $x$ and $y$ directions are now uncoupled, we can further simplify the system by only considering cooling in the $x$ direction. The state of the system is thus written in the the basis

$$
|J ; M\rangle\left|p_{x}\right\rangle
$$

The momentum is discretized to a grid of $\hbar k$ separation. Because the cooling light and doughnut mode have slightly different frequency we make the approximation that both the doughnut mode and the cooling light, as well as spontaneous emission, act on $p_{x}$ with quanta $\pm 1,0$ with the same unit $\hbar k$, i.e., we only take the detunings to have an effect on the internal state.

The contribution of the cooling beams, Eq. (4.5), now only contains the $x$ direction. With the definition of the $p_{x}$ grid with a fixed $k$ for all beams, the action of $\exp ( \pm i k x)$ is simply a shift \pm 1 of the state in the $p_{x}$ basis. Besides the two polarizations $\hat{\boldsymbol{\epsilon}}_{ \pm x}$ the cooling beams are still determined by two parameters, $\Delta_{c}$ and $\Omega_{c}$. For comparing the strength of the cooling light with $U_{d}$ we introduce the light shift parameter $U_{c}$, given by

$$
U_{c}=\frac{\hbar \Omega_{c}^{2}}{4 \Delta_{c}} .
$$

The Hamiltonian has become one dimensional,

$$
H\left(x, p_{x}\right)=\frac{p_{x}^{2}}{2 m}+H_{d}+H_{c}(x) .
$$

As we take our initial distribution for $p_{x}$ to be Gaussian with $\Delta p_{x}=10 \hbar k$, the grid is cut off at $\pm 30 \hbar k$. This leaves us with a state space of $12 \times 61=732$ dimensions. The density matrix has the squared number of elements, which is the reason for using state vector trajectories.

\section{A. Quantum trajectories}

Quantum trajectories are time-dependent state vectors of a dissipative system, which are solutions of a stochastic Schrödinger equation. This equation is designed such that the ensemble average of pure states reproduces the time-dependent density matrix that solves the master equation, Eq. (4.7), of the dissipative system [23].

In the present case, the actual simulations are performed using normalized state-diffusion trajectories [24]. Since we have discretized the momentum we must also discretize the effect of spontaneous emission on the atoms momentum. Assuming that the spontaneously emitted photons have circular $(\beta= \pm 1)$ or linear $(\beta=0)$ polarization the angular distribution functions of the emission are given by

$$
\begin{gathered}
g_{ \pm 1}(\hat{\mathbf{u}})=\frac{3}{8 \pi}\left(1-\frac{1}{2} \sin ^{2} \theta\right) \text { and } \\
g_{0}(\hat{\mathbf{u}})=\frac{3}{8 \pi} \sin ^{2} \theta .
\end{gathered}
$$

The corresponding distributions for the recoil $\mathbf{u}=\Delta \mathbf{p} / \hbar k$ in the $x$ direction are

$$
\begin{gathered}
g_{ \pm 1}\left(u_{x}\right)=\frac{9}{16}-\frac{3}{16} u_{x}^{2}, \\
g_{0}\left(u_{x}\right)=\frac{3}{8}+\frac{3}{8} u_{x}^{2} .
\end{gathered}
$$

It has been shown to be a good approximation to direct the spontaneous emission along one of the coordinate axes $x, y$, or $z$ [25]. For emission in the $x$ direction, the above distribution functions can be discretized after projection onto the the $x$ axis. The discrete probabilities follow uniquely from the requirement that the first moments $\overline{u_{x}}$ and $\overline{u_{x}^{2}}$ are the same as for the continuous distributions (5.6), for each one of the three independent polarizations $\beta= \pm 1,0$. This gives the probabilities $g_{\beta}^{\alpha}$, with $\alpha=\Delta p_{x} / \hbar k$,

$$
\begin{array}{cc}
g_{ \pm 1}^{ \pm 1}=\frac{3}{20}, & g_{ \pm 1}^{0}=\frac{7}{10}, \\
g_{0}^{ \pm 1}=\frac{1}{5}, & g_{0}^{0}=\frac{3}{5} .
\end{array}
$$

The change in the internal state is determined by the polarization $\beta$ of spontaneous emission.

The stochastic Schrödinger equation that generates the individual trajectories is given by

$$
\begin{aligned}
d|\psi(t)\rangle= & -\frac{i}{\hbar} H\left(x, p_{x}\right)|\psi(t)\rangle d t-\frac{\Gamma}{2}\left(\mathbf{Q} \cdot \mathbf{Q}^{\dagger}-2\langle\mathbf{Q}\rangle \cdot \mathbf{Q}^{\dagger}\right. \\
& \left.+\langle\mathbf{Q}\rangle \cdot\left\langle\mathbf{Q}^{\dagger}\right\rangle\right)|\psi(t)\rangle d t+\sqrt{\Gamma} \\
& \times \sum_{\alpha \beta}\left(Q_{\beta}^{\dagger}-\left\langle Q_{\beta}^{\dagger}\right\rangle\right) g_{\beta}^{\alpha} e^{i \alpha k x}|\psi(t)\rangle d \xi_{\beta}(t)
\end{aligned}
$$

with

$$
\left|d \xi_{\beta}(t)\right|=\sqrt{d t} \quad \text { and } \quad \overline{d \xi_{\beta}(t)}=0 .
$$

Equation (5.8) has three main parts. The first term is simply the Hamiltonian and it corresponds to the first term of Eq. (4.7). The second term is a deterministic, non-Hermitian term that simulates the first part of the second term of Eq. (4.7). The third term is a stochastic term that in second order approximately simulates the integral of Eq. (4.7). The stochastic increments $d \xi_{\beta}(t)$ fulfill the statistical requirements of Eq. (5.9) so that the first-order contribution of the stochastic terms vanish in the ensemble average. The nonlinear terms in Eq. (5.8) assure that the individual trajectories remain normalized. In the ensemble average $\overline{|\psi(t)\rangle\langle\psi(t)|}$ these nonlinear terms cancel and the density matrix $\rho(t)$ is recovered.

The statistical properties described by Eq. (5.9) still allow for a certain freedom in the actual choice of the values of $d \xi_{\beta}(t)$ per trajectory. Usually either real noise, where $d \xi_{\beta}(t)= \pm \sqrt{d t}$, or noise with a complex random phase is chosen. In earlier work, however, we have shown that for simulation of a single observable a more efficient choice can be made [6]. The complex phase of $d \xi_{\beta}(t)$ is then chosen in such a way that the individual realizations of the observable are not influenced to first order by the stochastic terms of Eq. (5.8). This way the individual realizations remain closer to the ensemble average than for noise with a random phase, so that an average over fewer trajectories is needed to converge to the exact result. Since we are interested in $\Delta p_{x}$, we adapt the phase of the noise to the observable $p_{x}^{2}$. One expects that 


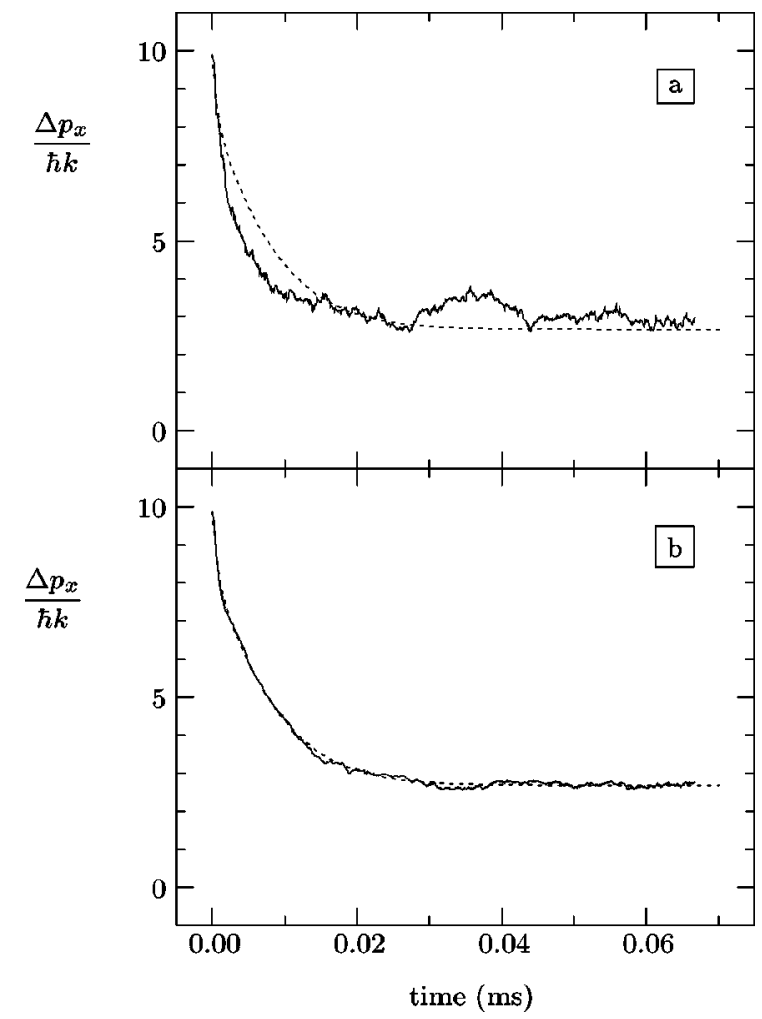

FIG. 7. Simulation of $\Delta p_{x}$ as a function of time using (a) random noise and (b) adaptive noise. For both methods 20 trajectories were averaged to obtain the solid curves. Both dotted curves are a fit of figure (b). The parameters are $\Delta_{c}=-5 \Gamma, U_{d}=330 E_{\mathrm{rec}}$, and $U_{c}=26 E_{\mathrm{rec}}$.

$\left\langle p_{x}\right\rangle \approx 0$ and that the noise in $\left\langle p_{x}^{2}\right\rangle$ is the main contributor to the noise of $\Delta p_{x}=\sqrt{\left\langle p_{x}^{2}\right\rangle-\left\langle p_{x}\right\rangle^{2}}$ per realization. For this reason we choose

$$
d \xi_{\beta}(t)= \pm i \frac{\left\langle Q_{\beta} p_{x}^{2}\right\rangle-\left\langle Q_{\beta}\right\rangle\left\langle p_{x}^{2}\right\rangle}{\left|\left\langle Q_{\beta} p_{x}^{2}\right\rangle-\left\langle Q_{\beta}\right\rangle\left\langle p_{x}^{2}\right\rangle\right|} \sqrt{d t}
$$

with equal probability for either sign. The benefits of using adaptive noise with respect to using noise with a random phase are illustrated in Fig. 7. For typical values of the parameters the time evolution of $\Delta p_{x}$ was simulated. Clearly, the average over just 20 trajectories has already converged for trajectories based on adaptive noise, in contrast to the average over trajectories based on randomly phased noise. The small amount of jittering noise that is still present in Fig. 7 (b) is due to the contribution of $\left\langle p_{x}\right\rangle^{2}$ to $\Delta p_{x}$. The individual realizations of $\left\langle p_{x}^{2}\right\rangle$ alone are perfectly smooth, although they slowly drift due to higher order contributions of the noise.

Figure 7(b) also shows the two time scales present in the simulation. In the initial stage the state of the system decoheres in terms of the correlations between the different momenta. In fact, the noise localizes the state in momentum space, causing a rapid decrease of $\Delta p_{x}$. This is actually an artifact resulting from the fact that we start with a coherent superposition of different momentum states instead of a classical mixture. However, after a relatively short time this superposition has decohered and from that moment on we are actually simulating a mixed state. The cooling takes place on a longer time scale. Although the initial value of $\Delta p_{x}$ is effectively less after decoherence, the final steady-state value may be expected to be the same in any case. The overall evolution turns out to be quite well described by a sum of two decaying exponents plus an offset, as can be seen from the dotted fit in Fig. 7. The two different time scales differ approximately by a factor 20 . In any case the fits are tight resulting in a good approximation of the final value of $\Delta p_{x}$ that we are interested in.

\section{B. Numerical results}

The parameters that are present in our approximate model are the effective light shift $U_{d}$, the detuning $\Delta_{c}$ and the Rabi frequency $\Omega_{c}$ of the cooling light, and the polarizations $\hat{\boldsymbol{\epsilon}}_{d}$ and $\hat{\boldsymbol{\epsilon}}_{ \pm x}$. In the simulation we let these parameters attain typical experimental values.

The doughnut polarization $\hat{\boldsymbol{\epsilon}}_{d}$ is taken to be linearly polarized $\pi_{y}$ (see Table I). With this choice $H_{d}$ is not just a light shift on the individual internal states $|J ; M\rangle$, but it also contains off-diagonal terms that slightly "reshuffle" the amplitudes of Zeeman substates with $\Delta M= \pm 2$ within the ground and excited states. However, we have found that there is no qualitative difference as compared to the results of simulations performed with a circularly polarized doughnut. For better comparison with the experimental results, most of which were also obtained using a linearly polarized doughnut, we present the simulation results for linear polarization.

The cooling polarization $\hat{\boldsymbol{\epsilon}}_{ \pm x}$ is taken to be $\sigma_{x}^{ \pm}$(see Table III). The detuning is fixed, $\Delta_{c}=-5 \Gamma$. For larger values of the detuning the simulations take more computation time. However, one must keep in mind that polarization-gradient cooling in free space, on a $J \rightarrow J+1$ transition, is known to work only for negative detunings of the cooling light. Therefore, cooling can in principle be expected only when $\left|\Delta_{c}\right|$ exceeds the additional light shift $U_{d}$, which reduces the effective detuning. In the simulations $U_{c}$ is varied by varying $\Omega_{c}$ within a typical experimental range.

The value of $U_{d}$ is taken as a fraction of $U_{\max }$. In the experiment some of the atoms will be on, or very near, the $z$ axis and will only be weakly influenced by the doughnut mode, i.e., they experience a small value of $U_{d}$. Other atoms will have a certain distance from the $z$ axis and will experience a larger fraction of $U_{\max }$. In our model, a simulation for a given value of $U_{d}$ therefore corresponds to a wave packet with a momentum spread of $10 \hbar k$ traveling at a certain distance from the doughnut-mode axis. The actual distribution of the atoms inside the doughnut mode can be viewed as a superposition of such wave packets. For comparison with the experiment the results of the simulation should be averaged over the atomic distribution inside the doughnut mode. For instance, if we take the parameters as mentioned in Sec. III B, where we measured a spatial spread of $6.5 \mu \mathrm{m}$, about $60 \%$ of the atoms experience a light shift larger than $600 E_{\mathrm{rec}}$. The dynamics during cooling, i.e., the change of $U_{d}$ when the atoms become cooler, can of course not be studied in this model.

In Fig. 8 the final values of $\Delta p_{x}$ have been plotted for different values of $U_{d}$ and $U_{c}$. The bottom curve, for which 


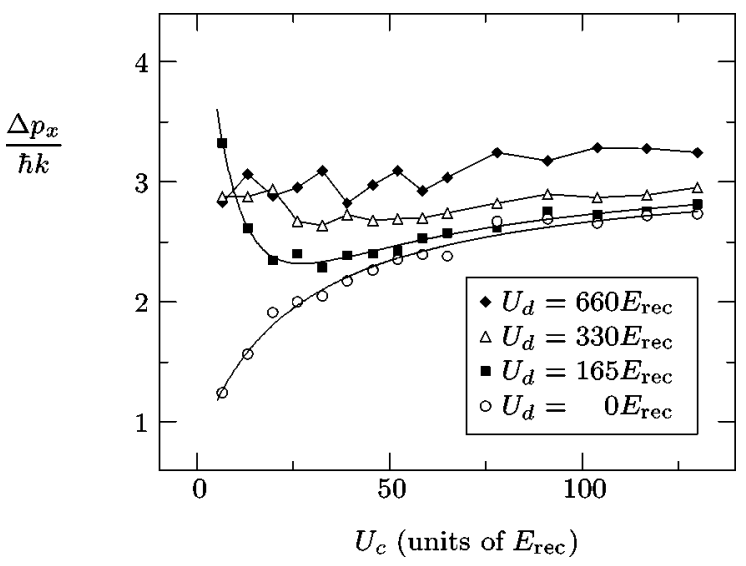

FIG. 8. Final value of $\Delta p_{x}$ as a function of different cooling intensities, with $\Delta_{c}=-5 \Gamma$, for different doughnut strengths. The curves serve as guides to the eye.

$U_{d}=0$, corresponds to cooling in free space. It displays the expected behavior: for decreasing cooling light intensities the final momentum spread approaches a value of slightly more than a single recoil. At very small values of $U_{c}$ the cooling is seen the break down and the momentum spread is much larger than a single recoil. For increasing values of $U_{c}$ the final temperature also increases as the saturation (and therefore the scattering rate) increases.

For higher values of $U_{d}$ the final value of $\Delta p_{x}$ asymptotically approaches the curve for $U_{d}=0$ as $U_{c}$ increases. For values $U_{c}<50 E_{\text {rec }}$, however, the behavior significantly deviates from cooling in free space. The final value of $\Delta p_{x}$ actually increases for decreasing $U_{c}$.

There appears to be a thresholdlike behavior of $U_{c}$ for a fixed value of $U_{d}$. Above the threshold, the cooling process is hardly affected by the doughnut potential. Below the threshold, the cooling process is disturbed by the presence of the doughnut and the final temperature is significantly higher than for cooling in free space. It seems that the cooling light has to outweigh the doughnut potential.

The coherent pumping of the cooling light is perturbed by the incoherent action of the doughnut mode. The light shifts induced by the doughnut mode, different for every $|J ; M\rangle$ sublevel, will give rise to a phase rotation of the amplitudes. If the pump rate of the cooling beams is much slower than this phase rotation, the coherences generated by the cooling light will decohere relatively rapidly. This will not only make the optical pumping less efficient, but it also gives rise to an offset of the excitation. The cooling light, inducing Rabi oscillations, will no longer periodically transfer all the population to the ground state as the amplitudes of $|2 ; M\rangle$ and $|3 ; M \pm 1\rangle$ will dephase due to the doughnut light shift. The excitation offset gives rise to an increase of spontaneous emission, heating the system. When the pump rate of the cooling beams is much faster than the phase rotation, the cooling process is hardly affected. The fact that far below threshold the cooling does not work and the fact that the temperature increases again for increasing values of $U_{c}$ far above threshold gives rise to a certain optimum value of $U_{c}$, for which the final temperature reaches a minimum.

The presence of a threshold is illustrated in Fig. 9. Below the threshold [see Fig. 9(a)] the final $p_{x}$ distribution, averaged over 20 trajectories, is asymmetrical and is even shifted

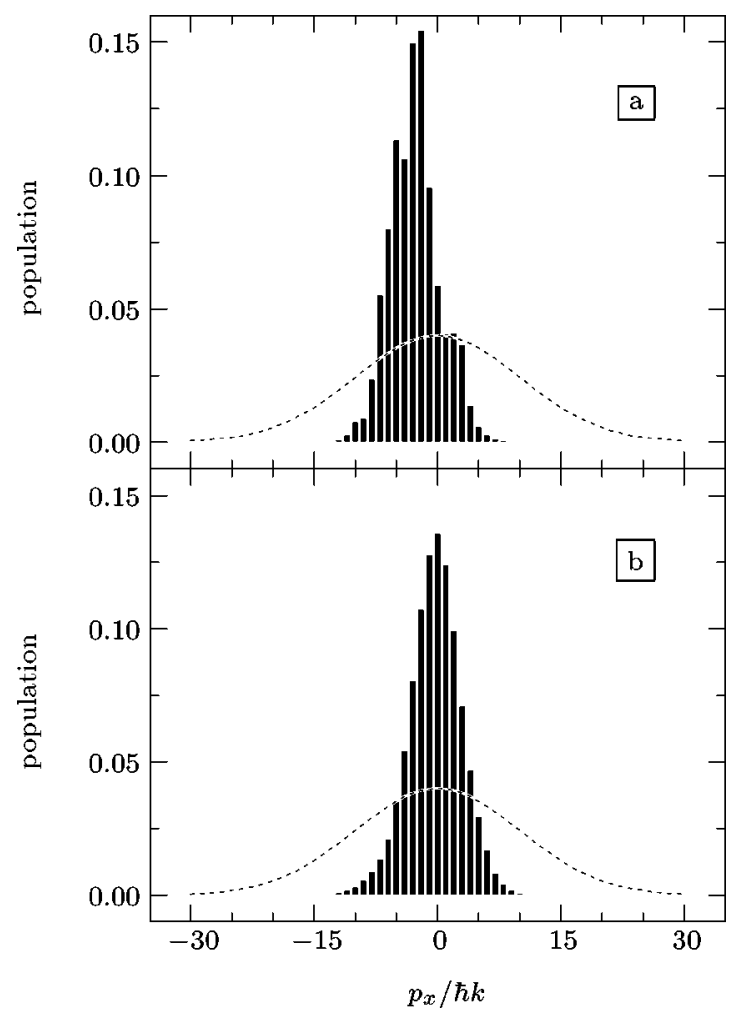

FIG. 9. Final $p$ distribution after cooling with parameters $\Delta_{c}=$ $-5 \Gamma, U_{d}=660 E_{\mathrm{rec}}$, and (a) $U_{c}=13 E_{\mathrm{rec}}$ and (b) $U_{c}=117 E_{\mathrm{rec}}$. The dotted curves represent the initial distribution.

from $p=0$. Above the threshold [see Fig. 9(b)] the final $p_{x}$ distribution is centered around $p=0$ and has a Gaussian shape.

A shift of $\overline{\left\langle p_{x}\right\rangle}$ due to the cooling process induces noise into the simulation because the adaptive method only stabilizes $\left\langle p_{x}^{2}\right\rangle$ and relies on the fact that $\left\langle p_{x}\right\rangle \approx 0$. The fact that $\overline{\left\langle p_{x}\right\rangle}$ may differ from zero below the threshold therefore explains the increased error of the simulation points in Fig. 8 for low values of $U_{c}$ and high values of $U_{d}$. Below threshold more than 20 trajectories are required to accurately calculate $\Delta p_{x}$.

Note that the restriction of minimal values for $\Delta_{c}$ and $U_{c}$ do not apply to the situation of cooling in free space. In the absence of an additional light shift potential the sublevels of both the ground and the excited state are fully degenerate. Hence the cooling beams have a uniform detuning, which may be arbitrarily small. Furthermore, the coherences between the different sublevels are preserved, apart from spontaneous decay, so there is no lower bound on the pump rate to be effective.

Also note that the light shift parameters $U_{d}$ and $U_{c}$ are light shifts with respect to a Clebsch-Gordan coefficient of one. In fact, every pair of $M$ sublevels experiences a different light shift, proportional to a Clebsch-Gordan coefficient less than one. Because these coefficients differ for different values of $M$, there is no single threshold value for $U_{c}$; there is rather a transition regime. This regime appears to be centered around the value for which $U_{c}$ becomes comparable in magnitude with $U_{d}$. The value of $U_{c}$ should be multiplied by a factor of two before comparison to take into account both counterpropagating cooling beams. 


\section{SUMMARY AND DISCUSSION}

The experimental data show that the guiding and focusing properties of the doughnut-mode potential, which we investigated in earlier work [4], are not altered in the presence of the cooling beams. The initial conditions for the cooling of the atom beam, like beam width and transverse temperature, can be controlled by the waist and intensity of the doughnut mode. On the other hand, the characteristic behavior of the cooling process is different for cooling in free space and cooling inside the doughnut mode. The final transverse temperature decreases for increasing cooling light intensities when the doughnut mode is present, in contrast to cooling in free space. For the investigated parameter range, the final temperature is seen to depend on the strength of the doughnut-mode potential. For weak potentials the minimum temperature remains close to that achieved without a doughnut mode present.

The experimental situation was simulated by a simplified 1D model with a spatially uniform light shift potential. The use of the adaptive noise method has been proven to be advantageous as it considerably reduces the computing time. Within this model, similar changes in the characteristic behavior are found in the numerical simulations within the same parameter range. We ascribe these changes to the existence of a threshold for the cooling light intensity; the light shift induced by the cooling light should exceed the light shift induced by the doughnut mode. The proposed mechanism is that the light shift induced by the doughnut alters the optical pumping of the cooling light. The pump rate of the cooling light diminishes, decreasing the cooling force, and an offset in the excitation causes heating due to an increased spontaneous emission rate.

Although a precise quantitative comparison between the experimental and numerical results is difficult because of the simplifications made, a few remarks can be made. As mentioned a major fraction of atoms inside the doughnut mode experience light shifts larger than $660 E_{\mathrm{rec}}$. This means that the uppermost curve in Fig. 8 describes a large portion of the atoms inside the doughnut mode. For large values of $U_{c}$ the value of $\Delta p_{x}$ with the additional light shift present is about $0.7 v_{\text {rec }}$ larger for these atoms when compared to cooling in free space. This agrees with the increase of $\Delta p_{x}$ observed in the experiment.

The experimental data show the presence of a hot and a cold component, not found in the simulation. The resolution of the momentum grid is not high enough to see such effects (see Figs. 3 and 9). However, the simulation shows that the velocity distribution changes shape when the doughnut-mode light shifts become stronger than those induced by the cooling light (see Fig. 9). This parallels the experimental observation, where the double Gaussian velocity distribution becomes a single Gaussian for strong doughnut modes.

As our simulations show, the higher temperatures found in the presence of the doughnut mode can already be understood as resulting from a spatially homogeneous light shift. Additionally, spatial variations may also cause heating. For example, at the slope of the doughnut mode potential the dipole force fluctuations [26] are enhanced. We expect that this additional contribution to the heating becomes important when the gradient of the doughnut-mode potential becomes comparable to the gradient of the light shift potential induced by the cooling light. This may explain the larger momentum widths obtained in the strong doughnut regime.

\section{ACKNOWLEDGMENT}

S.K. acknowledges financial support from the European Union (TMR Contract No. ERBFMBICT 961472).
[1] Ultracold Atoms and Bose-Einstein-Condensation, Vol. 7 of Trends in Optics and Photonics, edited by K. Burnett (Optical Society of America, Washington, DC, 1996).

[2] H. J. Lee, C. S. Adams, M. Kasevich, and S. Chu, Phys. Rev. Lett. 76, 2658 (1996).

[3] C. S. Adams, H. J. Lee, N. Davidson, M. Kasevich, and S. Chu, Phys. Rev. Lett. 74, 3577 (1995).

[4] S. Kuppens, M. Rauner, M. Schiffer, G. Wokurka, T. Slawinski, M. Zinner, K. Sengstock, and W. Ertmer, in Ultracold Atoms and Bose-Einstein Condensation, Vol. 7 of Trends in Optics and Photonics, edited by K. Burnett (Optical Society of America, Washington, DC, 1996), pp. 102-107.

[5] M. Schiffer, M. Rauner, S. Kuppens, K. Sengstock, and W. Ertmer, Appl. Phys. B (to be published).

[6] F. E. van Dorsselaer and G. Nienhuis, Eur. Phys. J. D 2, 175 (1998)

[7] A. E. Siegman, Lasers (University Science Books, Mill Valley, CA, 1986).

[8] W. L. Power, L. Allen, M. Babiker, and V. E. Lembessis, Phys. Rev. A 52, 479 (1995).

[9] H. Stecher, R. Deutschmann, W. Ertmer, and P. Zoller (unpublished).
[10] J. Nellessen, J. Werner, and W. Ertmer, Opt. Commun. 78, 300 (1990).

[11] E. Riis, D. Weiss, K. Moler, and S. Chu, Phys. Rev. Lett. 64, 1658 (1990).

[12] A. Scholz, M. Christ, D. Doll, J. Ludwig, and W. Ertmer, Opt. Commun. 111, 155 (1994).

[13] M. Schiffer, M. Christ, G. Wokurka, and W. Ertmer, Opt. Commun. 134, 423 (1997).

[14] The flux of injected atoms does not form a principal limitation. Recent experiments showed that it is also possible to omit the mirror and coaxially overlap the doughnut beam with the atom beam by sending it right through the 2D-MOT compression zone. In this case up to $60 \%$ of the atoms could be captured and guided by the doughnut beam.

[15] N. R. Heckenberg, R. McDuff, C. P. Smith, H. RubenszteinDunlop, and M. J. Wegener, Opt. Quantum Electron. 24, S951 (1992).

[16] M. T. Gale, M. Rossi, H. Schütz, P. Ehbets, H. P. Herzig, and D. Prongu, Appl. Opt. 32, 2526 (1993).

[17] M. Zinner, K. Sengstock, and W. Ertmer (unpublished).

[18] M. D. Hoogerland, H. F. P. de Bie, H. C. W. Beijerinck, E. J. D. Vredenbregt, and K. A. H. van Leeuwen, Phys. Rev. A 54, 3206 (1996). 
[19] D. Milic, M. D. Hoogerland, K. G. H. Baldwin, and R. E. Scholten, Quantum Semiclassic. Opt. 8, 629 (1996).

[20] R. E. Scholten, R. Gupta, J. J. McClelland, and R. J. Celotta, Phys. Rev. A 55, 1331 (1997).

[21] M. R. Williams, M. J. Bellanca, L. Liu, C. Xie, W. F. Buell, T. H. Bergeman, and H. J. Metcalf, Phys. Rev. A 57, 401 (1998).

[22] G. Nienhuis, P. van der Straten, and S.-Q. Shang, Phys. Rev. A 44, 462 (1991).
[23] H. J. Carmichael, An Open Systems Approach to Quantum Optics, Lecture Notes in Physics Vol. m18 (Springer-Verlag, Berlin, 1993).

[24] N. Gisin and I. C. Percival, J. Phys. A 25, 5677 (1992).

[25] Y. Castin, K. Berg-Sørensen, J. Dalibard, and K. Mølmer, Phys. Rev. A 50, 5092 (1994).

[26] J. Dalibard and C. Cohen-Tannoudji, J. Opt. Soc. Am. B 6, 2023 (1989). 